\title{
Integrated Droplet Routing in the Synthesis of Microfluidic Biochips*
}

\author{
Tao Xu and Krishnendu Chakrabarty \\ Department of Electrical and Computer Engineering \\ Duke University, Durham, NC 27708, USA \\ $\{\mathrm{tx}, \mathrm{krish}\} @ e e . d u k e . e d u$
}

\begin{abstract}
Microfluidic biochips are revolutionizing many areas of biochemistry and biomedical sciences. Several synthesis tools have recently been proposed for the automated design of biochips from the specifications of laboratory protocols. However, only a few of these tools address the problem of droplet routing in microfluidic arrays. These methods typically rely on post-synthesis droplet routing to implement biochemical protocols. Such an approach is not only time-consuming, but it also imposes an undue burden on the chip user. Moreover, post-synthesis droplet routing does not guarantee that feasible droplet pathways can be found for area-constrained biochip layouts; non-routable fabricated biochips must be discarded. We present a droplet-routing-aware automated synthesis tool for microfluidic biochips. Droplet routability, defined as the ease with which droplet pathways can be determined, is estimated and integrated in the synthesis flow. The proposed approach allows architectural-level design choices and droplet-routing-aware physical design decisions to be made simultaneously. We use a large-scale protein assay as a case study to evaluate the proposed synthesis method.
\end{abstract}

\section{Categories and Subject Descriptors \\ B.7.2 [Integrated Circuits]: Design Aids.}

\section{General Terms}

Algorithms, Performance, Design.

\section{Keywords}

Synthesis, routing, microfluidics, biochip.

\section{INTRODUCTION}

Microfluidics-based biochips offer exciting possibilities for high-throughput sequencing, parallel immunoassays, blood chemistry for clinical diagnostics, DNA sequencing, and environmental toxicity monitoring [1,2]. Prototype biochips have been developed and reported in the literature for enzymatic assays to detect glucose and lactate levels in blood [3], detection of explosives such as TNT [4], real-time monitoring of airborne particulate matter [5], synthesis-based gene sequencing [6], and protein crystallization [7].

Currently, most commercially-available biochips rely on continuous fluid flow in etched microchannels. Fluid flow is controlled either using micropumps and microvalves [8], or by electrokinetics [9]. An alternative category of microfluidic biochips relies on the principle of electrowetting-on-dielectric. Discrete droplets of nanoliter volumes can be manipulated in a "digital" manner on a two-dimensional electrode array. Hence this technology is referred to as "digital microfluidics”[10]. Since a digital microfluidic array is made up of

* This research was supported by the National Science Foundation under grant number IIS-0312352 and CCF-0541055.

Permission to make digital or hard copies of all or part of this work for personal or classroom use is granted without fee provided that copies are not made or distributed for profit or commercial advantage and that copies bear this notice and the full citation on the first page. To copy otherwise, or republish, to post on servers or to redistribute to lists, requires prior specific permission and/or a fee.

DAC 2007, June 4-8, 2007, San Diego, California, USA.

Copyright 2007 ACM 978-1-59593-627-1/07/0006 ...\$5.00. identical unit-electrodes, this system also offers dynamic reconfigurability, whereby groups of unit cells in a microfluidic array can be reconfigured to change their functionality during the concurrent execution of a set of bioassays.

As more bioassays and biomedical procedures are mapped on a microfluidic platform for concurrent execution, system functionality and design complexity are expected to increase steadily. Next-generation biochips are likely to be multifunctional and adaptive "biochemical processing” devices. For example, inexpensive biochips for clinical diagnostics offer high throughput with low sample volumes, and they integrate hematology, pathology, molecular diagnostics, cytology, microbiology, and serology onto the same platform. The emergence of such integrated, multifunctional, and reconfigurable platforms provides the electronic design automation community with a new application driver and market for research into new algorithms and design tools.

Several automated synthesis tools have recently been proposed for microfluidic biochips. These design automation methods address operation scheduling and module placement for digital microfluidics $[11,12,13,14,15,16]$, as well the design and optimization of routing channels in continuous-flow microfluidics [17]. However, none of these tools address the problem of droplet routing in digital microfluidic arrays. These methods typically rely on post-synthesis droplet routing to implement biochemical protocols; such an approach is not only time-consuming, but it also imposes an undue burden on the chip user. Moreover, post-synthesis droplet routing does not guarantee that feasible droplet pathways can be found for area-constrained biochip layouts; non-routable fabricated biochips must be discarded. Synthesis tools for digital microfluidics simply assume that the droplet routing time is negligible compared to microfluidic operation times, and that the droplet pathways can be trivially determined $[11,13,15,16]$.

However, as in the case of integrated circuits (ICs), routing complexity increases with an increase in the complexity and number of bioassay operations that are mapped to a digital microfluidics platform. The reconfigurability inherent in digital microfluidics leads to a large number of candidate droplet pathways, which must all be evaluated to determine the best synthesis solutions. Moreover, unlike permanently etched microchannels in continuous-flow microfluidic chips, the droplet pathways in digital microfluidics are reconfigurable and can be activated or deactivated dynamically.

Some methods for post-synthesis droplet routing have recently been proposed [18, 19, 20], but these methods suffer from the need to explicitly evaluate a large number of candidate routes. Moreover, while recent advances in digital microfluidics have significantly reduced microfluidic operations times [21], droplet routing time has not decreased proportionately. Similar to the increased importance of interconnect length in integrated circuits, droplet pathway lengths are now an important consideration for biochip system synthesis. Therefore, to reduce droplet routing complexity and to obtain synthesis results that best predict bioassay operation times, droplet routing needs to be integrated in the synthesis flow.

In this paper, we propose a droplet-routing-aware unified synthesis method that combines architectural synthesis with 
droplet-routing-aware physical design. Droplet routability, defined as the ease with which droplet pathways can be determined, is estimated and integrated in the synthesis flow. The proposed approach allows architectural-level design choices and droplet-routing-aware physical design decisions to be made simultaneously. We use a large-scale protein assay as a case study to evaluate the proposed synthesis method.

The rest of the paper is organized as follows. Section 2 provides an overview of digital microfluidic biochips. In Section 3 we discuss related prior work on automated synthesis tools and post-synthesis droplet routing for digital microfluidic biochips. In Section 4, we introduce a new criterion of evaluating droplet routability for a synthesized design, and incorporate it into the overall synthesis flow. Section 5 uses a large-scale protein assay to evaluate the proposed synthesis method. Finally, conclusions are drawn in Section 6.

\section{DIGITAL MICROFLUIDIC BIOCHIPS}

The microfluidic biochips discussed in this paper are based on the manipulation of nanoliter droplets on a two-dimensional electrode array using the principle of electrowetting [10]. A unit cell in the array includes a pair of electrodes that acts as two parallel plates. The bottom plate contains a patterned array of individually controlled electrodes, and the top plate is coated with a continuous ground electrode. A droplet rests on a hydrophobic surface over an electrode. It is moved by applying a control voltage to an electrode adjacent to the droplet and, at the same time, deactivating the electrode just under the droplet. This electronic method of wettability control creates interfacial tension gradients that move the droplets to the charged electrode. Using the electrowetting phenomenon, droplets can be moved to any location on a two-dimensional array.
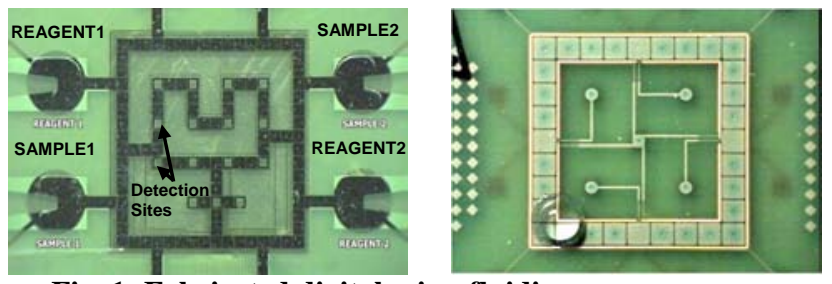

Fig. 1: Fabricated digital microfluidic arrays.

By varying the patterns of control voltage activation, many fluid-handling operations such as droplet merging, splitting, mixing, and dispensing can be executed in a similar manner. For example, mixing can be performed by routing two droplets to the same location and then turning them about some pivot points [21]. The digital microfluidic platform offers the additional advantage of flexibility, referred to as reconfigurability, since fluidic operations can be performed anywhere on the array. Droplet routes and operation scheduling result are programmed into a microcontroller that drives electrodes in the array. In addition to electrodes, optical detectors such as LEDs and photodiodes are also integrated in microfluidic arrays to monitor colorimetric bioassays [22]

\section{RELATED PRIOR WORK}

Recent years have seen growing interest in the automated design and synthesis of microfluidic biochips [11,12,13,14,15,16,17]. One of the first published methods for biochip synthesis decouples high-level synthesis from physical design [11]. It is based on rough estimates for placement costs such as the areas of the microfluidic modules. These estimates provide lower bounds on the exact biochip area, since the overheads due to spare cells and cells used for droplet transportation are not known a priori. However, it cannot be accurately predicted if the biochip design meets system specifications, e.g., maximum allowable array area and upper limits on assay completion times, until both high-level synthesis and physical design are carried out. [12]

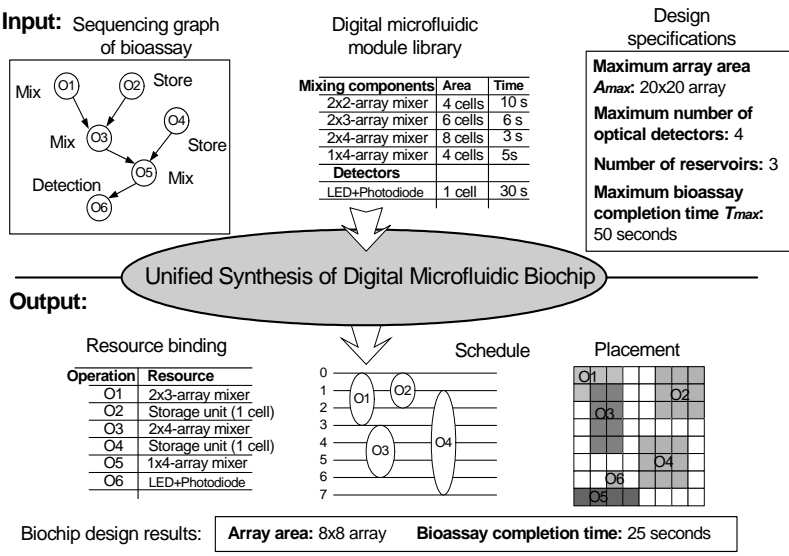

Fig. 2. An example illustrating system-level synthesis [12].

proposed a unified system-level synthesis method for microfluidic biochips based on parallel recombinative simulated annealing (PRSA), which offers a link between these two steps. This method allows users to describe bioassays at a high level of abstraction, and it automatically maps behavioral descriptions to the underlying microfluidic array.

The design flow is illustrated in Fig. 2. First, the different bioassay operations (e.g. mixing and dilution), and their mutual dependences are represented using a sequencing graph. Next, a combination of simulated annealing and genetic algorithms are used for unified resource binding, operation scheduling, and module placement. A chromosome is used to represent each candidate solution, i.e., a design point. In each chromosome, operations are randomly bound to resources. Based on the binding results, list scheduling is used to determine the start times of operations, i.e., each operation starts with a random latency after its scheduled time. Finally, a module placement is derived based on the resource binding and scheduling information.

A weighted sum of area- and time-cost is used to evaluate the quality of the design. The design is improved through a series of genetic evolutions based on PRSA. It generates an optimized schedule of bioassay operations, the binding of assay operations to resources, and a layout of the microfluidic biochip.

The top-down synthesis flow described above unifies architecture level design with physical level module placement. However, it suffers from two drawbacks. For operation scheduling, it is assumed that the time cost for droplet routing is negligible, which implies that droplet routing has no influence on the operation completion time. While generating physical layouts, the synthesis tool in [12] provides only the layouts of the modules and it leaves droplet routing pathways unspecified. The assumption of negligible droplet transportation times is valid works for small microfluidic arrays. However, for large arrays and for biochemical protocols that require several concurrent fluidic operations on-chip, the droplet transportation time and routing complexity are non-trivial.

Recent work on automated biochip design has also included post-synthesis droplet routing $[18,20]$. These methods can reduce droplet transportation time by finding optimal routing plans for a synthesized biochip. However, the effectiveness of such methods is limited by the synthesis results, i.e., the placement of microfluidic modules often determines the droplet pathways that lead to minimum droplet transportation time. For example, if we need to route a droplet between two modules that are 10 electrodes away from each other, then it is not possible to reduce the droplet transportation time to less than that needed to move a droplet by a distance equal to 10 electrodes. Since droplet pathways are dynamically reconfigurable, the number of 
feasible droplet pathways can be very high, leading to considerable computation time for a droplet routing tool.

Automated droplet routing is also essential to ensure that digital microfluidic biochips are adopted by the user community of biochemists. In view of the increasingly complex liquid handling protocols being implemented in digital microfluidics, there is a pressing need for automating the operations. Currently, all the assays are programmed by hand and it is very complex to program droplet operations for more than two assays performed in parallel on-chip. Particularly, the end user should not be burdened with any programming tasks and the operation of the biochips should ideally be completely transparent, wherein the user only has to specify the assay protocol and input the liquid samples.

It is therefore necessary to synthesize biochip designs that make droplet routing easier. Moreover, due to advances in microfluidic module design (smaller feature sizes, improved materials, etc.), the fluidic operation times are decreasing steadily [21]. However, the droplet transportation times are not decreasing at the same pace. As a result, routing times must be considered during operation scheduling and in the calculation of assay completion times. For the synthesis results derived from the methods proposed in [12], the impact of droplet routing on assay completion time might be significant, and the upper limit on assay completion time might be violated. In such scenarios, the biochip design will no longer correctly implement the desired biochemical procedures. Also, if a synthesized design is not routable, either the chip must be discarded or time-consuming resynthesis must be carried out. To avoid such occurrences, we have to anticipate the availability of routing paths during synthesis. Therefore, droplet routing must be included in the synthesis flow for digital microfluidics.

\section{DROPLET-ROUTING-AWARE SYNTHESIS}

Droplet-routing methods can be viewed as being either anticipatory, i.e., anticipate the routability (defined qualitatively as the ease of droplet routing) of the synthesized biochip and design the system to be easily routable, or based on post-synthesis routing to find the efficient droplet pathways.

The focus of this paper is on droplet-routing-aware design, whereby we attempt to provide a guaranteed level of routability for every interdependent module pair that needs to be connected to each other. Instead of finding efficient droplet pathways after synthesis, we attempt to achieve high-routability mapping of bioassay protocols to the microfluidic array. In this section, we propose a new method to incorporate droplet routing in the PRSA-based synthesis flow for defect-tolerant microfluidic biochips developed in [12].

\subsection{Droplet-Routability Estimation}

For a synthesized biochip, the droplet-routability of a route between two modules is quantified in terms of the length, measured by the number of electrodes, of the droplet transportation path. Droplet-routability is evaluated in terms of the average length of all the droplet pathways for the complete chip. Also, we have to control the maximum length of droplet paths. Large values for the maximum path length lead to long routing times, e.g., more than $5 \%$ of the module operation time, which can have the undesirable consequence of having to halt an assay temporarily until the droplets are routed to their destinations. Moreover, long routing pathways are liable to be blocked by obstacles, i.e., intermediate modules. For example, in Fig. 3, all routing pathways from $M_{1}$ to $M_{4}$ are blocked by $M_{2}$ and $M_{3}$, therefore droplet routing is not feasible for this design. Note that guard-ring cells are used to avoid inadvertent mixing and they cannot be used for routing. Synthesized designs with large values for the maximum droplet path length suffer from a high probability of being non-routable. Based on the above considerations, we adopt the

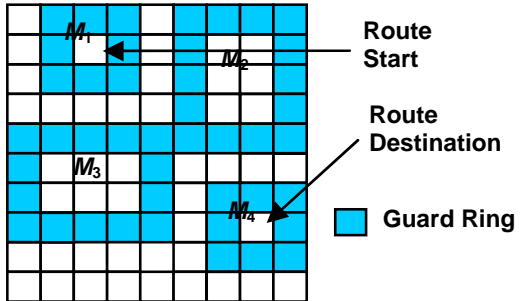

Fig. 3: An example of a non-routable interdependent pair.

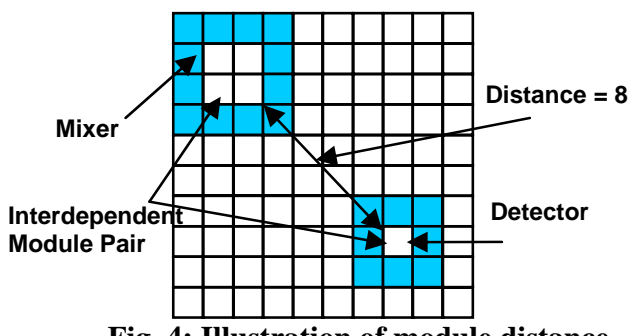

Fig. 4: Illustration of module distance.

maximum droplet path length as a parameter for evaluating routability of a synthesized biochip.

A straightforward technique to derive the routability information is to carry out post-synthesis routing to generate an actual routing plan. However, this approach adds to the computational burden of the synthesis tool. In particular, if a routing plan involving all the droplets on the array is generated for each chromosome in the PRSA-based unified synthesis method, the overall synthesis time will be overwhelming due to the large number of chromosomes and evolution steps in the synthesis flow. Moreover, since we only care about the final synthesis result, we need to reduce the effort spent to generate route plans for the intermediate designs. Therefore, we adopt simple estimates of routability, instead of precisely calculating droplet routes at each step.

The module distance $M_{i j}$ is defined as the length of the shortest path between two interdependent modules $M_{i}$ and $M_{j}$, assuming that there are no obstacles between them. By interdependent module, we refer to module pairs where the operation of one module depends on the operation of the other module. For example, if optical detection is to be carried out for a mixed droplet, then the optical detector and the mixer are interdependent. Note that in many cases, two interdependent modules may not be able to operate in successive time-steps; for example, a mixed droplet may have to wait for a few cycles since the detector may be busy processing another detection step when the mixing is finished. In such cases, a storage unit is needed, and we consider the storage unit and the detector as interdependent modules. So are the mixer and the storage unit.

Thus droplets are routed only between interdependent modules. The module distance is calculated for each interdependent module pair. Although the module distance $M_{i j}$ may not be exactly the same as the shortest path length, especially if there are obstacles in the form of other modules on the array, $M_{i j}$ is still a good estimate of routability between $M_{i}$ and $M_{j}$.

Note that in some scenarios, the location of two interdependent modules may overlap on the array. In this case, we set the corresponding module distance to be zero. Since our goal is to guarantee the routability of modules in the synthesized biochip, we adopt the average module distance (over all interdependent modules) as a design metric. Similarly, we adopt maximum module distance to approximate the maximum length of droplet manipulation and use it for routability estimation. For each chromosome considered in the PRSA-based synthesis flow, we calculate the average and maximum module distance.

Next we incorporate routability in the PRSA-based unified 


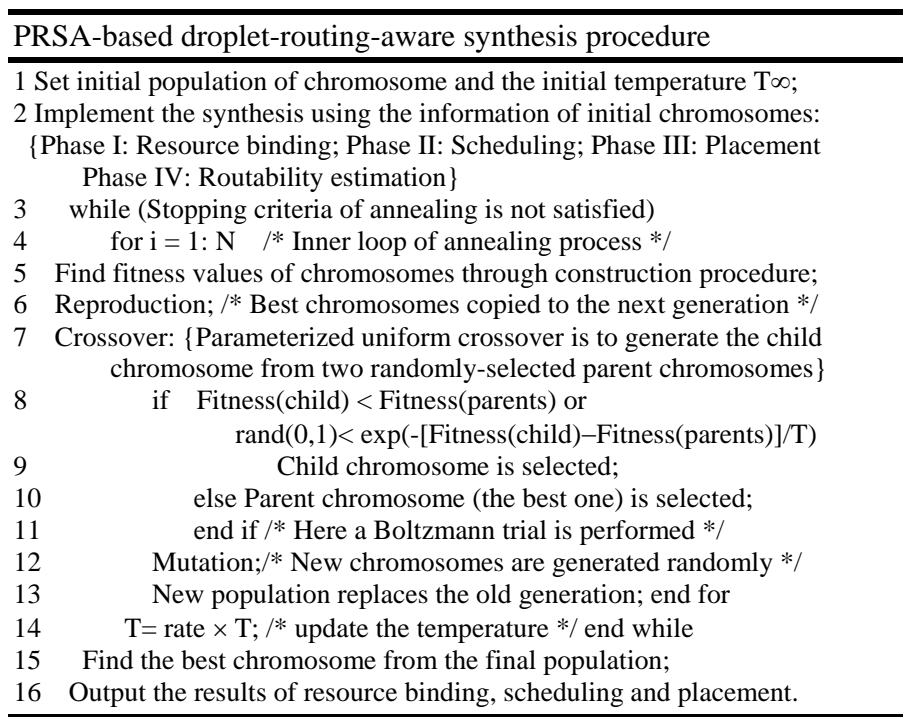

Fig. 5: Pseudocode for the PRSA-based droplet-routing-aware synthesis procedure.

synthesis method. Synthesis results with high routability values are more likely to lead to simple and efficient droplet pathways. To find such designs, we incorporate the above two metrics into the fitness function by a factor that can be fine-tuned according to different design specifications to control the PRSA-based procedure. The pseudocode for the droplet-routing-aware unified synthesis method is shown in Figure 5. Candidate designs with low routability are discarded during evolution. Thus, the synthesis procedure guarantees that the routing complexity is reduced for the synthesized biochip, while meeting constraints on array size, bioassay processing time, and defect-tolerance [12].

\subsection{Routing Time Cost and Assay Completion Time}

Next we discuss the impact of routing time cost on bioassay completion time. Here we use the route planning method of [20] to find an efficient route plan for each interdependent pair. The time cost due to the need for droplet transportation is calculated and added to the operation time for the first module in the interdependent module pair. Next the schedule is adjusted based on the modified operation time.

There are two possible scenarios that can arise when the schedule is adjusted. In the first scenario, despite the increased operation time, the fluidic operation can be accommodated in its designated time interval due to the availability of slack or unoccupied time slots in the schedule. In this case, the schedule can simply rely on the available slack or unused time interval for droplet routing. In the second scenario, operations are scheduled so tightly that there is not enough slack available for routing. In this paper, we deal with this problem by adding an extra time slot for routing. As a result, the schedule result is "relaxed" and the completion time is increased. Note that in relaxing the schedule, the ordering of the start times of operations is not changed, therefore, the change in the schedule has no impact on other aspects of synthesis, namely resource binding, module placement, and defect-tolerance. The updated assay completion time includes the routing time cost and reflects the actual time needed for executing the biochemical protocol on the synthesized biochip.

\section{EXPERIMENTAL EVALUATION}

In this section, we evaluate the droplet-routing-aware synthesis method by using it to design a biochip for a real-life protein assay.

Recently, the feasibility of performing a colorimetric protein assay on a digital microfluidic biochip has been successfully demonstrated

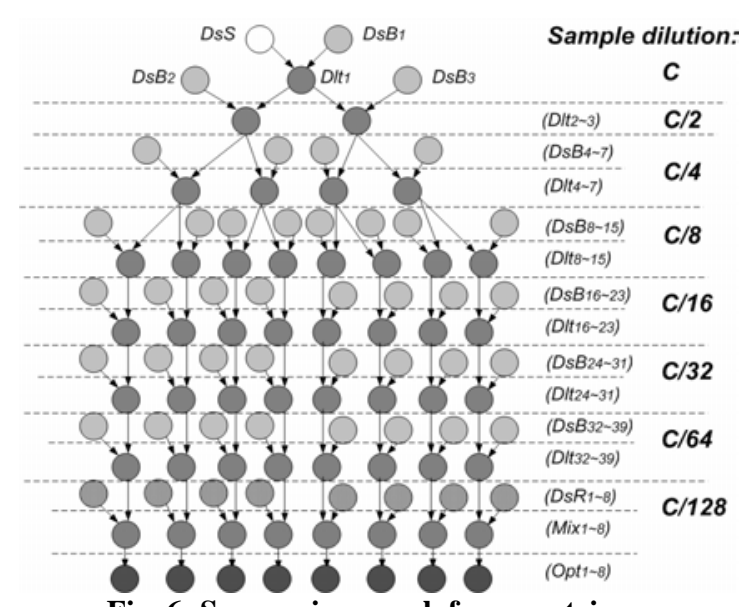

Fig. 6: Sequencing graph for a protein assay.

Table 1. Experimentally-characterized module library for synthesis.

\begin{tabular}{|c|l|c|}
\hline Operation & \multicolumn{1}{|c|}{ Resource } & Time (s) \\
\hline DsS;DsB; DsR & On-chip reservoir/dispensing port & 7 \\
\hline \multirow{4}{*}{ Dlt } & 2x2-array dilutor & 12 \\
\cline { 2 - 3 } & 2x3-array dilutor & 8 \\
\cline { 2 - 3 } & 2x4-array dilutor & 5 \\
\cline { 2 - 3 } & 4-electrode linear array dilutor & 7 \\
\hline \multirow{4}{*}{ Mix } & 2x2-array mixer & 10 \\
\cline { 2 - 3 } & 2x3-array mixer & 6 \\
\cline { 2 - 3 } & 2x4-array mixer & 3 \\
\cline { 2 - 3 } & 4-electrode linear array mixer & 30 \\
\hline Opt & LED+Photodiode & N/A \\
\hline Storage & Single cell & \\
\hline
\end{tabular}

[23]. Based on the Bradford reaction [20], the protocol for a generic droplet-based colorimetric protein assay is as follows. First, a droplet of the sample, such as serum or some other physiological fluid containing protein, is generated and dispensed into the biochip. Buffer droplets, such as $1 \mathrm{M} \mathrm{NaOH}$ solution, are then introduced to dilute the sample to obtain a desired dilution factor $(D F)$. This on-chip dilution is performed using multiple hierarchies of binary mixing/splitting phases, referred to as the interpolating serial dilution method [10]. The mixing of a sample droplet of protein concentration $C$ and a unit buffer droplet results in a droplet with twice the unit volume, and concentration $C / 2$. Splitting this large droplet results in two unit-volume droplets of concentration $\mathrm{C} / 2$ each. Continuing this step in a recursive manner using diluted droplets as samples, an exponential dilution factor of $D F=2^{N}$ can be obtained in $N$ steps. After dilution, droplets of reagents, such as Coomassie brilliant blue G-250 dye, are dispensed into the chip, and they mix with the diluted sample droplets. Next the mixed droplet is transported to a transparent electrode, where an optical detector (e.g., a LED-photodiode setup) is integrated. The protein concentration can be measured from the absorbance of the products of this colorimetric reaction using a rate kinetic method [20]. Finally, after the assay is completed, all droplets are transported from the array to the waste reservoir. A sequencing graph model can be developed from the above protocol for a protein assay $(D F=128)$, as shown in Figure 6. There are a total of 103 nodes in one-to-one correspondence with the set of operations in a protein assay, where $D s S, D s B_{i}(i=1, \ldots, 39)$, and $D s R_{i}(i=1, \ldots, 8)$ represents the generation and dispensing of sample, buffer and reagent droplets, respectively.

In addition, Dlt $_{i}(i=1, \ldots, 39)$ denotes the binary dilution (including mixing/splitting) operations, $\operatorname{Mix}_{i}(i=1, \ldots, 8)$ represents the mixing of diluted sample droplets, and reagent droplets; $\operatorname{Opt}_{i}(i=$ $1, \ldots, 8)$ denotes the optical detection of the droplets. Until the fourth step of a serial dilution, all diluted sample droplets are retained 
in the microfluidic array. After that stage, for each binary dilution step, only one diluted sample droplet is retained after splitting, while the other droplet is moved to the waste reservoir.

The basic operations for the protein assay have been implemented on a digital microfluidic biochip [10, 20]. Experiments indicate that the dispensing operation takes 7 seconds [11]. The operation times of various mixers have been found to be different [10]. A binary dilution operation can also be easily implemented by mixing of sample droplet followed by droplet splitting. Absorbance of the assay product can be measured using an integrated LED-photodiode setup. Experiments indicate this absorbance measurement takes 30 seconds [23]. The microfluidic module library for a protein assay is shown in Table 1 . A total of 122 interdependent module pairs must be routed for this protocol. Clearly, the large number of droplet transportation operations in this protocol makes it difficult for a biochemist user or a post-synthesis design tool to determine transportation paths. We also need to specify some design parameters for the biochip to be synthesized. Different design specifications can be determined based on user needs and manufacturing constraints.

To evaluate the proposed routing-aware synthesis method, we first apply it to an example in which we set the maximum microfluidic array size to be 100 cells, and the maximum allowable completion time for the protein assay to be 400 seconds. We assume that there is only one on-chip reservoirs/dispensing port available for sample fluids, but two such ports for buffer fluids, two for reagent fluids, and one for waste fluids. Finally, we assume that at most four optical detectors can be integrated into this biochip.

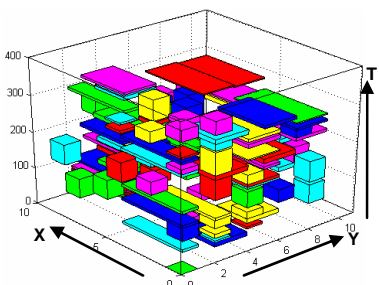

(a)

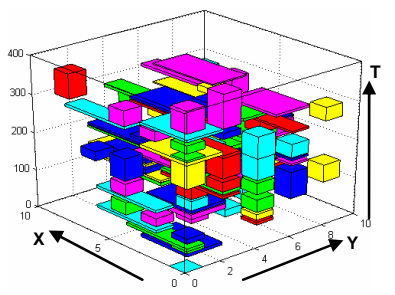

(b)
Fig.7: A 3-D model illustrating the synthesis results: (a) routing-oblivious method of [12]; (b) the proposed method.

We first use the routability-oblivious PRSA-based algorithm from [12] to find a desirable solution for the protein assay that satisfies design specifications. The solution thus obtained yields a biochip design with a $10 \times 10$ microfluidic array, an assay completion time of 377 seconds, a maximum module distance of 14 electrodes, and an average distance of 3 electrodes. Next we use the droplet-routing-aware synthesis method using the procedure of Fig. 5. The procedure yields a biochip design with a $10 \times 10$ microfluidic array, a completion time of 378 seconds, a maximum module distance of 7 electrodes, and an average distance of 1 electrode. The computation time for the routability-oblivious and routing-aware methods for the protein assay are 15 minutes and 16 minutes, respectively on a 1.86 GHz Pentium-M PC with $1 \mathrm{G}$ of RAM.

We illustrate the synthesis results, i.e., assay operation schedule and module placement, using a 3-D box model shown in Fig. 7. Each microfluidic module is represented as a 3-D box, the base of which denotes the rectangular area of the module and the height denotes the time-span of the corresponding assay operation. The projection of a 3-D box on the $\mathrm{X}-\mathrm{Y}$ plane represents the placement of this module on the microfluidic array, while the projection on the Z-axis (time axis) represents the schedule of the assay operation. Note that the synthesis results determine the locations of integrated optical detector. Transparent electrodes for optical detection are used in the microfluidic array.

Although the two designs have comparable area- and time- cost, the

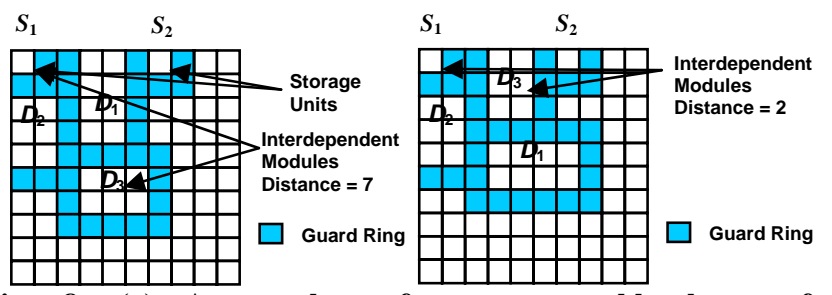

Fig. 8: (a) A snapshot of a non-routable layout from routing-oblivious synthesis (time instant 297s) (b) Corresponding layout in routing-aware synthesis (time instant 299s).

routing-aware synthesis method leads to a $50 \%$ reduction in the average and maximum module distance. This indicates a significant improvement of routability and reduction of the time-cost for carrying out droplet-routing. To verify this improvement, we applied the post-synthesis routing method of [20] to find efficient droplet pathways routing for both layouts. We find that while routing-aware synthesis easily leads to a feasible routing plan, the layout for the routing-oblivious result is not routable, i.e., no pathway is available for certain droplet manipulations. Fig. 8(a) shows a snapshot of the layout for the routing-oblivious result taken at time instant 297s. In this snapshot, a droplet is to be routed from the storage unit $S_{1}$ to dilutor $D_{3}$, which is located 7 electrodes away in the routing-oblivious layout. However, as shown in Fig. 8(a), there is no pathway available for routing due to the compact layout and large module distance. In contrast, in the layout derived from the routing-aware synthesis procedure, since the average module distance is minimized, $D_{3}$ is placed next to $S_{1}$ and the droplet pathway can be trivially determined.

Thus we can see that without violating constraints on time- and area-cost, the routing-aware method carefully arranges interdependent modules to be close to each other. Therefore, it ensures that droplet pathways can be determined with a high probability. On the other hand, the routing-oblivious method only aims at meeting satisfying constraints on time- and area-cost. As a result, the interdependent modules are likely to be segregated by other modules when routing-oblivious synthesis is employed; a consequence of this is that routing solutions cannot be obtained. Without a careful arrangement of modules, routing-oblivious synthesis can find feasible routes only if the area constraint is fairly loose, thereby enough chip area is available to create droplet pathways. As a result, time- and area-cost are compromised and the design specifications might not be met.

We examine this issue as follows. We first synthesize the protein assay under a set of design specifications using both the routing-oblivious and routing-aware synthesis methods. The pool of design specifications is defined by the Cartesian product of a set of time-cost limits $\left(T=\left\{T_{1}, T_{2}, T_{3}, \ldots, T_{n}\right\}\right)$ and a set of area-cost limit $(A=$ $\left.\left\{A_{1}, A_{2}, A_{3}, \ldots, A_{n}\right\}\right)$. Therefore, each synthesized chip $G_{i j}$ corresponds to a point $\left(T_{i,}, A_{j}\right)$. For each synthesized chip, we check if it is routable. A point $\left(T_{\mathrm{i},}, A_{\mathrm{j}}\right)$ is referred to as a feasibility boundary point if there are no other points $\left(T_{m}, A_{n}\right)$ such that $G_{i j}$ is routable and $T_{m}<$ $T_{i}, A_{n}<A_{j}$. A feasibility frontier is defined by connecting all the feasibility boundary points. Therefore, the feasible design region is defined by the area above the feasibility frontier. Here we set $T=\{320$, $340,360, \ldots, 440\}$ and $A=\{60,70,80, \ldots, 180\}$ and carry out both the routing-oblivious and routing-aware synthesis (the unit of $T$ is seconds while the unit of $A$ is measured in terms of the number of electrodes). The feasibility frontier is derived for both methods, as plotted in Fig. 9. Note that in finding the feasibility frontier, we fix a time limit and search for the minimum chip area for which a routable synthesis result is available.

As shown in Fig. 9, routing-aware synthesis leads to a lower feasibility frontier and a larger feasible design region. For tight time limits, e.g., 320s, the routing-aware method achieves a routable 


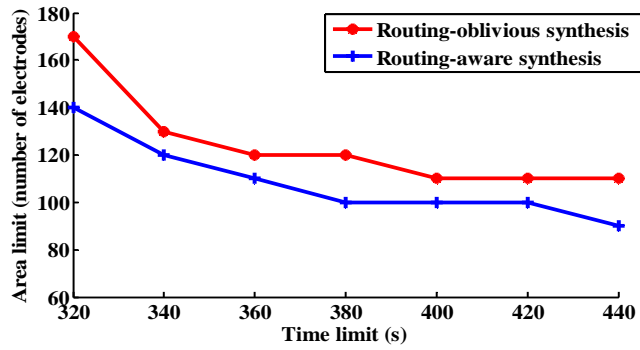

Fig. 9: Feasibility frontier and feasible design region for [12] and the routing-aware synthesis method.

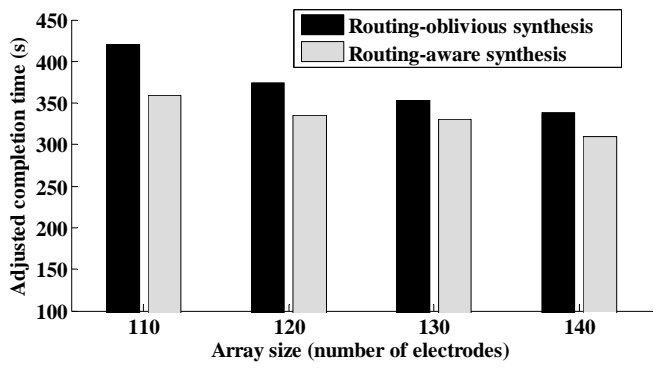

Fig. 10. Assay completion times (with droplet transportation time included) for [12] and for the proposed routing-aware synthesis method.

synthesis result with less than 140 electrodes, while the routing-oblivious method fails when the area limit is lower than 170 electrodes. On the other hand, for a fixed array size, e.g., 110 electrodes, routing-aware synthesis leads to a much lower assay completion time (less than 360s) than the routing-oblivious method (between 380s and 400s). The improvement becomes more significant when routing time is considered and added to the assay completion time. In addition to assay-time reduction, routing-aware synthesis allows us to reduce chip area, and thereby the product cost for disposable and reusable biochips.

Next we carry out post-synthesis routing for all the routable synthesis results corresponding to the feasible layouts and use schedule relaxation as defined in Section 4 to derive the adjusted completion time. We add the droplet transportation time to the assay completion time in each case. The results are shown in Fig. 10. These results show that that in addition to providing a greater range of feasible design points, routing-aware synthesis leads to lower assay completion times. The minimization of the assay completion time is essential for environmental monitoring applications where sensors can provide early warning. Real-time response is also necessary for surgery and neo-natal clinical diagnostics. Finally, biological samples are sensitive to the environment and to temperature variations, and it is difficult to maintain an optimal clinical or laboratory environment on chip. To ensure the integrity of assay results, it is therefore desirable to minimize the time that samples spend on-chip before assay results are obtained. Increased throughout also improves operational reliability. Long assay durations imply that high actuation voltages need to be maintained on some electrodes, which accelerate insulator degradation and dielectric breakdown, reducing the number of assays that can be performed on a chip during its lifetime. Therefore, the minimization of assay processing time using droplet-aware synthesis is very promising for the emerging biochips market.

\section{CONCLUSIONS}

We have presented a droplet-routing-aware automated synthesis tool for microfluidic biochips. Droplet routability, defined as the ease with which droplet pathways can be determined, has been estimated and integrated in the synthesis flow. This approach improves upon biochip design automation tools that rely on post-synthesis droplet routing to implement biochemical protocols. The proposed method increases the likelihood that feasible droplet pathways can be for area-constrained biochip layouts. It allows architectural-level design choices and droplet-routing-aware physical design decisions to be made simultaneously. We have demonstrated the advantages of this approach using a large-scale protein assay based on the Bradford reaction. We have also shown that the integration of droplet routing in the biochip synthesis flow leads to lower bioassay completion times and smaller chip area.

\section{REFERENCES}

[1] T. H. Schulte et al., "Microfluidic technologies in clinical diagnostics", Clinica Chimica Acta, vol. 321, pp. 1-10, 2002.

[2] V. Srinivasan et al., "Clinical diagnostics on human whole blood, plasma, serum, urine, saliva, sweat, and tears on a digital microfluidic platform”, Proc. $\mu T A S$, pp.1287-1290, 2003.

[3] A. G.-Elie et al., "Design of a subcutaneous implantable biochip for monitoring of glucose and lactate” IEEE Sensors Journal, 5(3), 345-355, 2005.

[4] V. K. Pamula et al., "A droplet-based lab-on-a-chip for colorimetric detection of nitroaromatic explosives”, Proc. IEEE MEMS Conf., 2005.

[5] R. B. Fair et al., "Integrated chemical/biochemical sample collection, pre-concentration, and analysis on a digital microfluidic Lab-on-a-Chip platform," in Lab-on-a-Chip: Platforms, Devices, and Applications, L.A. Smith and D. Sobek, eds., Proc. SPIE, vol. 5591, pp. 113-124, 2004.

[6] D. W. Deamer and M. Akeson, "Nanopores and nucleic acids: prospects for ultrarapid sequencing," Trends Biotechnol. vol. 18, pp. 147-151, 2000.

[7] M. van der Woerd et al., "The promise of macromolecular crystallization in microfluidic chips", Journal of Structural Biology, vol. 142, pp. 180-187, 2003.

[8] E. Verpoorte and N. F. De Rooij, "Microfluidics meets MEMS", Proc. IEEE, vol. 91, pp. 930-953, 2003.

[9]Y. Wang et al., "Composable behavioral models and schematic-based simulation of electrokinetic Lab-on-a-Chip Systems”, IEEE Trans. CAD, vol. 25, pp. 258-273, Feb. 2006.

[10] R. B. Fair et al., "Electrowetting-based on-chip sample processing for integrated microfluidics”, Proc. IEDM, pp. 32.5.1-32.5.4, 2003.

[11] F. Su and K. Chakrabarty, "Architectural-level synthesis of digital microfluidics-based biochips”, Proc. ICCAD, pp. 223-228, 2004.

[12] F. Su and K. Chakrabarty, "Unified high-level synthesis and module placement for defect-tolerant microfluidic biochips”, Proc. DAC, pp. 825-830, 2005.

[13] F. Su and K. Chakrabarty, "Module placement for fault-tolerant microfluidics-based biochips", ACM Transactions on Design Automation of Electronic Systems, vol. 11, pp. 682-710, 2006.

[14] W. Hwang et al., "Automated design of 'pin-constrained' digital microfluidic arrays for lab-on-a-chip applications”, Proc. DAC, pp. 925-930, 2006.

[15] C.-L.Yuh et al., "Placement of digital microfluidic biochips using the T-tree formulation”, in Proc. DAC, pp. 931-934, 2006

[16] A. J. Ricketts et al., "Priority scheduling in digital microfluidics-based biochips.” Proc. DATE, pp. 329-334, 2006.

[17] A. J. Pfeiffer et al., "Synthesis of multiplexed biofluidic microchips", IEEE Trans. CAD, vol. 2, pp.321-333, Feb. 2006.

[18] K. F. Böhringer, "Modeling and controlling parallel tasks in droplet-based microfluidic systems." IEEE Trans. CAD, vol. 25, pp.329-339, Feb. 2006

[19] E. J. Griffith et al., "Performance characterization of a reconfigurable planar array digital microfluidic system," IEEE Trans. CAD, vol. 25, pp. 340-352, Feb. 2006.

[20] F. Su et al, "Droplet routing in the synthesis of digital microfluidic biochips”, Proc. DATE, pp. 323-328, 2006.

[21] P. Y. Paik et al., "Rapid droplet mixers for digital microfluidic systems", Lab on a Chip, vol. 3, pp. 253-259, 2003.

[22] J. Zeng and T. Korsmeyer, "Principles of droplet electrohydrodynamics for lab-on-a-chip”, Lab on a Chip, pp. 265-277, 2004.

[23] V. Srinivasan et al., "Protein stamping for MALDI mass spectrometry using an electrowetting-based microfluidic platform”, Proc. SPIE, vol. 5591, pp. 26-32, 2004. 